\title{
A Realistic Computational Model of the Local Circuitry of the Cuneate Nucleus
}

\author{
Eduardo Sánchez ${ }^{1}$, Senén Barro ${ }^{1}$, Jorge Mariño ${ }^{2}$, and Antonio Canedo ${ }^{2}$ \\ 1 Grupo de Sistemas Intelixentes (GSI) \\ Departamento de Electrónica e Computación, Facultade de Físicas, \\ Universidade de Santiago de Compostela, \\ 15706 Santiago de Compostela, Spain \\ \{elteddy, elsenen\}@usc.es \\ http://elgsi.usc.es/index.html \\ 2 Departamento de Fisioloxía, Facultade de Medicina, \\ Universidade de Santiago de Compostela, \\ 15706 Santiago de Compostela, Spain \\ \{fsancala, xmarinho\}@usc.es
}

\begin{abstract}
Intracellular recordings obtained under cutaneous and lemniscal stimulation show that the afferent fibers can establish excitatory and inhibitory synaptic connections with cuneothalamic neurons [5] In addition, distinct types of recurrent collaterals with the capability of either exciting or inhibiting both cuneothalamic neurons and interneurons were also discovered [6]. With these data we have generated hypothesis about which circuits are implicated and also developed realistic computational models to test the hypothesis and study the cuneate properties [17,18. The results show that the cuneate could perform spatial and temporal filtering and therefore detect dynamic edges.
\end{abstract}

\section{Introduction}

The cuneate nucleus is a structure of the somato-sensory system that is located within the brain stem, in the most rostral area of the spinal chord. With regard to its spatial organization, three areas can be distinguished: caudal, middle and rostral [2]. The middle zone, where the experimental work reported in this study has been carried out, can in turn be divided into two areas [7]: a core (central region), and a shell (peripheral region). The core is basically made up of cuneothalamic neurons, which are also referred to as projection or relay neurons. The shell is basically made up of local neurons, also known as interneurons or non-thalamic projection neurons.

The main cuneate inputs originate from primary afferent (PAF) and corticocuneate fibers. The PAFs establish synaptic contact with projection cells and interneurons [7]. The great majority of the projection neurons in the middle cuneate send their axons to the contralateral ventroposterolateral thalamic (VPL) nucleus via the medial lemniscus [112:7].

To study the neuronal properties of the middle cuneate, intracellular and whole-cell techniques have recently been used in vivo [131234]. It was found 
that cuneothalamic neurons possess two modes of activity: oscillatory and tonic [4]. During deep anaesthesia, the cuneothalamic neurons show oscillatory bursting activity. This behavior changes to the tonic mode when injecting depolarizing currents or stimulating the peripheral receptive fields. A low-threshold calcium current $\left(I_{T}\right)$ and a hyperpolarization-activated cation current $\left(I_{h}\right)$ have been postulated to explain the bursting mode of activity [4]. All these data were considered to develop realistic computational models of the cuneate neurons 1516.

In this paper we shifted our focus to provide a model of the local circuitry of the cuneate. The presentation is organized as follows: (1) a brief description of the experimental methods, (2) a summary of the results, (3) an explanation of the hypothesis derived by these data, (4) a description of the computational model, (5) the simulation results, and (6) a final discussion.

\section{Experimental Methods}

All experiments conformed to Spanish guidelines (BOE 67/1998) and European Communities council directive (86/609/EEC), and all efforts were made to minimize the animals used. Data were obtained from cats $(2.3-4.5 \mathrm{~kg})$, which were anesthetized ( $\alpha$-cloralosa, $60 \mathrm{mg} / \mathrm{Kg}$, i.p.), paralyzed (Pavulon, $1 \mathrm{mg} / \mathrm{kg} / \mathrm{h}, \mathrm{i} . \mathrm{v}$ ) and placed under artificial respiration [4].

Cuneothalamic projection cells were identified as antidromically activated by medial lemniscus stimulation according to standard criteria, including the collision test as well as confirmation that the critical interval in the collision was not due to soma refractoriness 4]. For cutaneous stimulation, a plate of $20 \mathrm{mmx} 5 \mathrm{~mm}$ was specifically designed to place 6 electrode pairs. Electrodes were $3 \mathrm{~mm}$ separated from each other. With this organization a total length of $15 \mathrm{~mm}$ could be stimulated, which its beyond the average size of the cutaneous receptor fields.

\section{Experimental Results}

Experiments were performed to analyze neural responses under cutaneous and lemniscal stimulation. In the former case, we are looking for primary afferent effects. In the later case, we are interested in recurrent collateral influences.

1. Cutaneous stimulation

Stimulation over the center of a cuneothalamic receptive field originates excitatory postsynaptic potentials (Fig. 11). After stimulation of points located far away from the center, inhibitory postsynaptic potentials were found.

2. Lemniscal stimulation

This kind of protocol has induced cuneothalamic responses that consist on a pair of spikes. The first one appears to be an antidromic spike. The second one is clearly synaptic. The explanation can be found on the existence of recurrent collaterals from neighbour neurons that were previously activated by the lemniscal stimulation. 

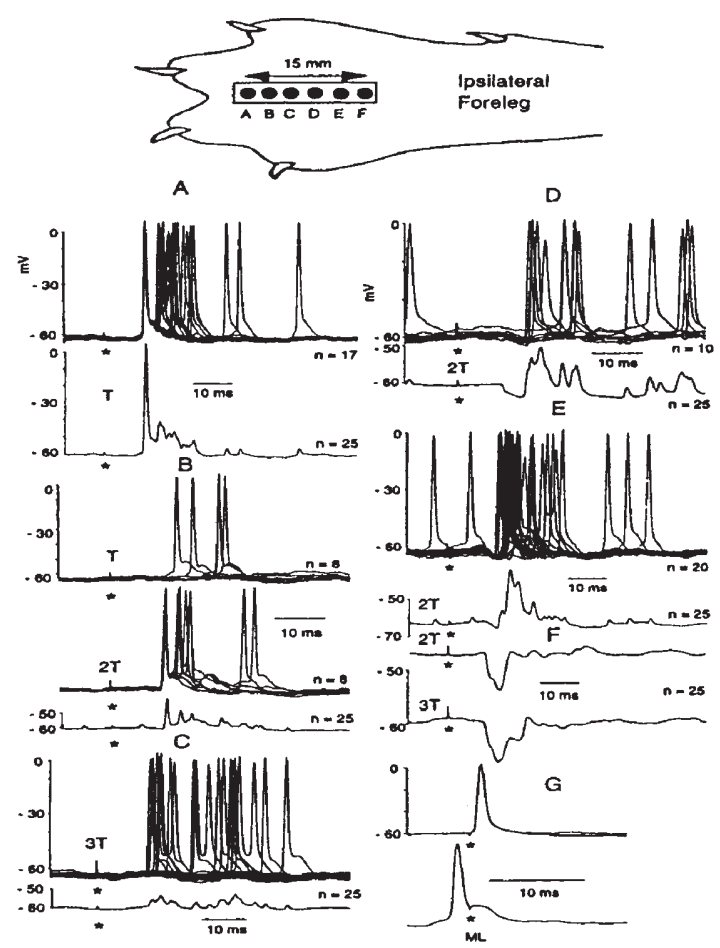

Fig. 1. Recordings under cutaneous stimulation. In the upper part, a drawing pictures the stimulation points in the cat's pow. In A, excitatory responses with lowest latency and stimulation intensity $(\mathrm{T})$ are shown. In points $\mathrm{B}$ and $\mathrm{C}$, higher stimulation $(2 \mathrm{~T}$ in $\mathrm{B} ; 3 \mathrm{~T}$ in $\mathrm{C}$ ) is required to obtain a similar response, although with higher latency. $\mathrm{D}$ and E show inhibitory-excitatory sequences. Finally, point F, in the periphery, shows only inhibitory responses under stimulation. In $G$, we show the antidromic identification (adapted from Canedo and Aguilar, 2000a).

Lemniscal stimulation can also induce inhibition over cuneothalamic neurons (Fig. 2). The inhibition response appears after the antidromic spike and can be explained based on the existence of inhibitory recurrent collaterals between cuneothalamic neurons.

\section{Some Hypothesis}

The experimental data shown above is the basis of the following hypothesis:

- Afferent excitation and inhibition. If cuneothalamic neurons have centersurround receptive fields, the cuneate can detect edges. This functional feature can be found also in other sensory systems.

- Recurrent lateral inhibition. This mechanism allows a neuron to inhibit the response of less activated neighbours. This effect is only observed over neigh- 
bour cells with non-overlapped receptive fields. In this way, only neurons with higher afferent input in a certain region are finally activated.

- Recurrent lateral excitation. Its function could be to synchronize the activity of neighbouring cells with overlapped receptive fields. In this way, a focus of activity would be generated to improve both signal localization and signal quality.

\section{The Computational Model}

\subsection{Neuron Level}

The multi-compartmental approach was used to model the neurons [14]. The membrane potential for each compartment is computed by using the following expression:

$$
C \frac{\partial V}{\partial t}=-I_{m}-I_{\text {syn }}-I_{\text {inject }}-\frac{\left(V^{\prime}-V\right)}{R_{a}^{\prime}}-\frac{\left(V^{\prime \prime}-V\right)}{R_{a}^{\prime \prime}}
$$

where $\mathrm{C}$ is the membrane capacitance, $I_{m}$ the sum of the ionic currents, $I_{\text {syn }}$ the synaptic current $I_{\text {inject }}$ the electrical stimulation, $R_{a}$ the axial resistance, and $\frac{\left(V^{\prime}-V\right)}{R_{a}^{\prime}}$ and $\frac{\left(V^{\prime \prime}-V\right)}{R_{a}^{\prime \prime}}$ denotes the axial current between each compartment and its adjacent ones.

We have used the following compartments and ionic currents:

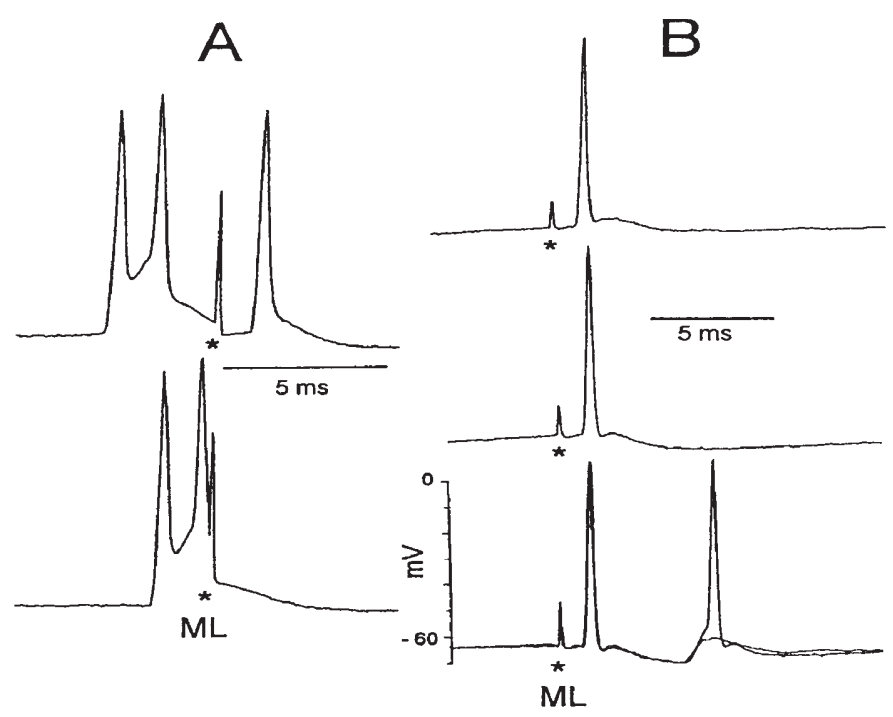

Fig. 2. Recordings under lemniscal stimulation and possible effects of inhibitory recurrent connections. In A, the antidromic identification is shown. In B, an appropriate level of stimulation at the medial lemniscus can induce an inhibition with a rebound that generates spike (lower picture in B) (Adapted from Canedo et al., 2000b). 
- Primary afferents. Two compartments for soma and axon. The membrane current is the sum of a fast sodium current and a delayed rectifier potassium current. Therefore, $I_{m}=I_{N a}+I_{K}$.

- Cuneothalamic cells. Three compartments for soma and two dendritic branches. The most remarkable ionic channels are a high-threshold calcium current $I_{L}$, a calcium-activated potassium current $I_{a h p}$, a hyperpolarizationactivated cationic current $I_{h}$, and a low-threshold calcium current $I_{T}$.

- Interneurons. Three compartments for soma, axon and dentritic tree. The ionic currents are very similar to those dicussed for cuneothalamic cells with two major differences: (1) a new slow potassium current $I_{A}$, and (2) the substitution of $I_{a h p}$ with other calcium-dependent potassium current $I_{C}$.

\subsection{Membrane Level}

All ionic currents were described by following the Hodgkin-Huxley model 9 . The mathematical description of all this currents can be found in 1719 .

\subsection{Synaptic Level}

To describe the conductance associated with the synaptic current we have used the alpha function. This conductance is the reasonable of generating postsynaptic potentials after the activation of dendritic receptors by appropriate neurotransmitters 10. The mathematical expression is:

$$
g_{\text {syn }}(t)=\alpha \frac{t-t^{\prime}}{\tau} e^{-\frac{t-t^{\prime}-\tau}{\tau}}
$$

where $\alpha$ is a constant, t' the initial time, and $\tau$ the time constant. The synaptic conductance value will be zero if the presynaptic potential is less than a certain threshold and it will be equal the alpha function above such threshold. The threshold represents the amount of neurotransmitter needed to activate the postsynaptic receptors.

The conductance value $g_{\text {syn }}$ determines the dynamics and the value of the synaptic current $I_{\text {syn }}$ :

$$
I_{\text {syn }}(t)=g_{\text {syn }}(t)\left(V-V_{\text {syn }}\right)
$$

where $V_{\text {syn }}$ denotes the resting potential of the postsynaptic membrane. In our model we introduce both excitatory and inhibitory connections. We assume the later being glutamatergic and the former being gabaergic. The excitatory connections are modeled by setting $V_{\text {syn }}$ to a positive value. The inhibitory connections are setting to a negative value.

\subsection{Simulation Tools}

For the simulations we have used Neuron. The simulation uses an integration method developed by Hines [8], which it is a fast procedure that optimizes performance by combining the advantages of Crank-Nicholson and Euler methods. The machine used for the simulations was a PC with a $600 \mathrm{Mhz}$ Pentium III processor. 


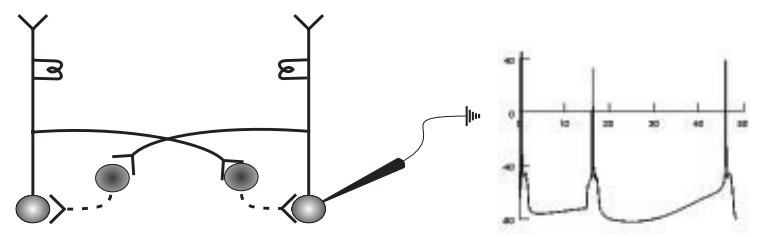

Fig. 3. Lemniscal stimulation and the effect of the inhibitory recurrent connections.

\section{Simulation Results}

We have developed two different circuits. The first one was used to validate th circuit with some experimental data. This circuit was implemented with $2 \mathrm{cu}-$ neothalamic projection neurons, 4 interneurons and 7 sensory receptors. With this configuration we can describe the complete receptive fields of both cuneothalamic cells. We only present here the simulation that shows the dynamics of the inhibitory recurrent collaterals under lemniscal stimulation. We have applied a $2 \mathrm{~ms}$ pulse and observe the response of two projection cells that show inhibitory recurrent connections between them. These connections were configured with a constant time $\tau=3$, maximum synaptic conductance $g_{\text {sin }}^{\max }=0.1$ and synaptic potential $V_{\text {syn }}=-90$. These values were adjusted to obtain the most similar response between the simulations and the experimental data. Figure 3 shows the neuronal activity after stimulation in $t=15 \mathrm{~ms}$. Current stimulation initially generates an antidromic spike and, afterwards a synaptic induced inhibition. This inhibition induces an hyperpolarization that activates the cationic current $I_{h}$, which in turns depolarizes the membrane and, finally, triggers the activation of a low-threshold calcium current $I_{T}$. The calcium potential generates a sodium spike. We believe that this mechanism can explain the experimental data.

In the second circuit we have developed a simplified cuneate with 7 cuneothalamic neurons and 14 sensory receptors. To simplify the computational cost we have replaced the interneurons with appropriate inhibitory gabaergic connections over the cuneothalamic neurons. The connections were set up in such a way that the contribution of the inhibitory periphery is more than the contribution of the excitatory center. We have analyzed in other simulations not shown here, that this is the only configuration that allows edge detection. In Fig. 4 we show the circuit and the stimulus chosen to test it. Receptors R6 and R7 were activated less than the others. What we get is that only Ct1 was activated. Although Ct4 shows a strongest postsynaptic activity than its neighbours, its activity is not higher enough to elicit response. This behaviour can be explained by the effect of the recurrent lateral inhibition exerted from Ct1 over its neighbours. With such a configuration, the cuneate would be able to detect the most significant events that appear in the environment.

And finally we want to introduce a new temporal filtering mechanism as a hypothesis. By considering some experimental evidence we believe that autoinhibitory connections can exist in the cuneate and it can perform a type of 


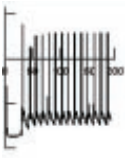

ㄷ1

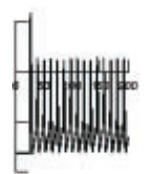

(B1)

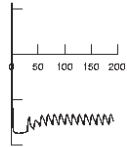

C12

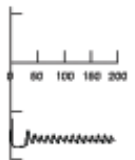

C고

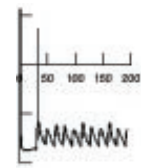

(6)

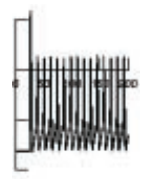

(2)

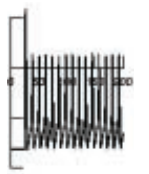

R3

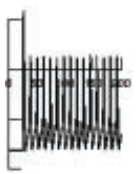

R4

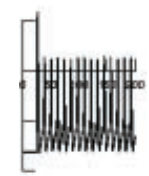

(85)

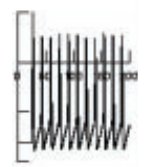

R6

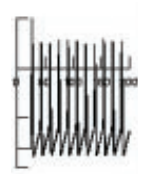

B7

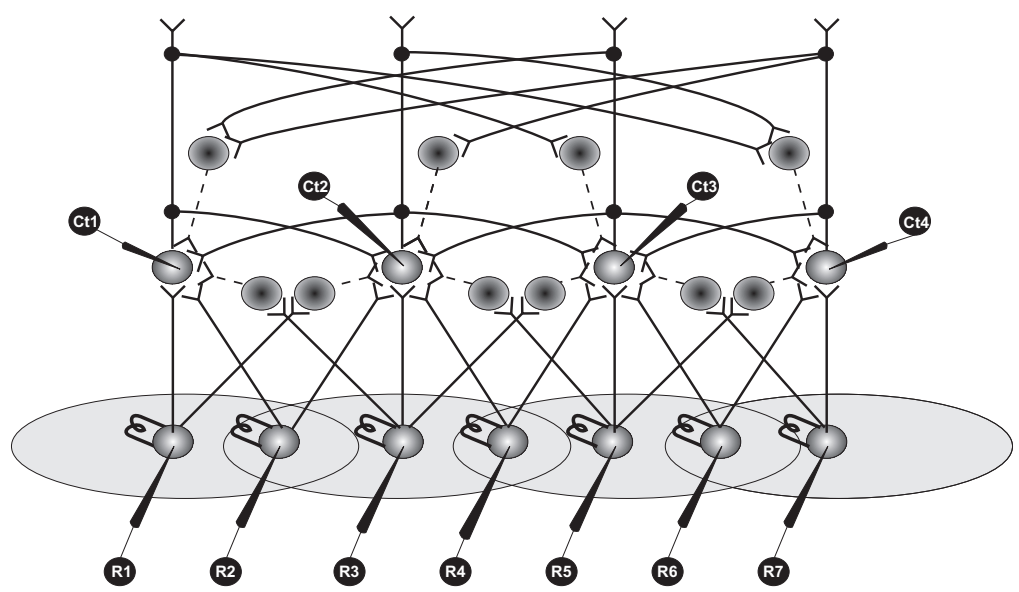

Fig. 4. Maximum edge detection. The concurrent activity of both afferent and recurrent connections permits the detection of the maximun edge in the left side of the stimuli.

temporal filtering process. What would happen if we integrate such a mechanism within the circuitry developed so far (Fig. 4)? The results are shown in Fig. 5 in which we have used a uniform stimulus. We observe that edge detection is performed first and, after a certain time interval, the cuneothalamic neurons stop firing. If the stimulus persists, the neurons enter in an oscillatory cycle that could serve as a pattern to signal that no relevant change is present in the environment.

\section{Discusion}

The main idea behind this study is that the cuneate performs a basic preprocessing of afferent input signals that consists on detecting changes from the outside. Detecting the edges of an stimulus is an example of detecting stimuli with different intensity patterns applied over the skin. The maximum edge detection is an 


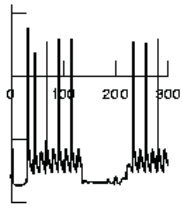

(6)

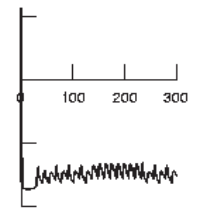

C12

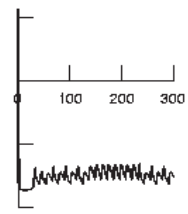

C+3

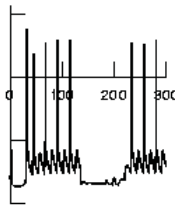

Ct4

Fig. 5. Temporal filtering and response of cuneothalamic neurons under a persistent stimulus with large surface. The new circuit can detect edges and the temmporal filtering mechanism is the responsible of generating oscillatory activity to signal the presence of a persitent stimuli.

example of detecting differences between selected edges. Detection of dynamic activity is an example of detecting changes in time. All these functional features reinforce the idea that the nervous system is designed to detect changes in the environment and to discard those signals that do not contribute with relevant information [1].

\section{Acknowledgments}

We would like to thank to Laboratorios de Neurociencia y Computación neuronal (LANCON), the environment in which this work has been developed.

\section{References}

1. Barlow H. B.: The coding of sensory messsages. In "Current problems in animal behaviour". Cambridge University Press. Thorpe W. H. and Zangwill O. L. (Eds.) (1961) 330-360

2. Berkley Karen J., Badell Richard J., Blomqvist A., Bull M.: Output Systems of the Dorsal Column Nuclei in the cat. Brain Research Review. Vol. 11 (1986) 199-225

3. Canedo, A.: Primary motor cortex influences on the descending and ascending systems. Progress in Neurobiology. Vol. 51 (1997) 287-335

4. Canedo A., Martinez L., Mariño J.: Tonic and bursting activity in the cuneate nucleus of the chloralose anesthetized cat. Neuroscience. Vol. 842 (1998) 603-617

5. Canedo A., Aguilar J.: Spatial and cortical influences exerted on cuneothalamic and thalamocortical neurons of the cat. European Journal of Neuroscience. Vol. 12 2 (2000) 2513-2533

6. Canedo A., Aguilar J., Mariño J.: Lemniscal recurrent and transcortical influences on cuneate neurons. Neuroscience. Vol. 972 (2000) 317-334

7. Fyffe Robert E., Cheema Surindar S., Rustioni A.: Intracelular Staining Study of the Feline Cuneate Nucleus. I. Terminal Patterns of Primary Afferent Fibers. Journal of Neurophysiology. Vol. 56.5 (1986) 1268-1283

8. Hines M.: A program for simulation of nerve equations with branching geometries. International Journal of Biomedical Computation. Vol. 24 (1989) 55-68 
9. Hodgkin A., Huxley A., Katz B.: Measurements of current-voltage relations in the membrane of the giant axon of Loligo. Journal of Physiology (London). Vol. 116 (1952) 424-448

10. Jack J. J. B., Redman S. J.: The propagation of transient potentials in some linear cable structures. J. of Physiology (London). Vol. 215 (1971) 283-320

11. Kuypers H. G. J. M., Tuerk J. D.: The distribution of the cortical fibers within the nuclei cuneatus and gracilis in the cat. J. Anat. Lond. Vol. 98 (1964) 143-162

12. Mariño J., Martínez L., Canedo A.: Coupled slow and delta oscillations between cuneothalamic and thalamocortical neurons in the chloralose anesthetized cat. Neuroscience Letters. Vol. 219 (1996) 107-110

13. Mariño J., Martínez L., Canedo A.: Sensorimotor integration at the dorsal column nuclei. News In Physiological Sciences. Vol. 14 (1999) 231-237

14. Rall W.: Theoretical significance of dendritic tree for input-output relation. In Neural Theory and Modeling. Stanford University Press, Stanford. Reiss, R. F. (Ed). (1964) 73-97

15. Sánchez E., Barro, S., Canedo, A., Martinez, L., Mariño, J.: Computational simulation of the principal cuneate projection neuron. Workshop P̈rinciples of Neural Integration: Instituto Juan March de Estudios e Investigaciones (1997). Madrid.

16. Sánchez E., Barro, S., Canedo, A., Martinez, L., Mariño, J.: A computational model of cuneate nucleus interneurons. Eur. J. Neurosci. Vol. 10. 10 (1998) 402

17. Sánchez E., Barro S., Mariño J, Canedo A., Vázquez P.: Modelling the circuitry of the cuneate nucleus. In Lecture Notes in Computer Science. Volume I. Springer Verlag. Mira J. and Sánchez Andrés J. V. (Eds). (1999) 73-85.

18. Sánchez E., Barro S., Mariño J, Canedo, A.: Selección de información y preprocesamiento de señales somatosensoriales en los núcleos de las columnas dorsales. Revista de Neurología. Viguera C. (Ed). (1999) 279.

19. Sánchez E.: Modelos del cuneatus: de la biología a la computación. Ph. D. Thesis. Santiago de Compostela. (2000). 